\title{
Evidence That Disinhibition Is Associated with a Decrease in Number of Vesicles Available for Release at Inhibitory Synapses
}

\author{
Veronica A. Ledoux and Catherine S. Woolley \\ Department of Neurobiology and Physiology and Northwestern University Institute for Neuroscience, Northwestern University, Evanston, Illinois 60208
}

We used three-dimensional reconstruction from serial electron micrographs to investigate two structural changes that could underlie estrogen-induced disinhibition of hippocampal CA1 pyramidal cells: a decrease in the number of inhibitory inputs per neuron and/or a change in inhibitory boutons that could limit GABA release. We analyzed 373 boutons forming 510 inhibitory synapses in estrogentreated and control animals. Our results show that estrogen specifically decreases the number of synaptic vesicles adjacent to the presynaptic membrane of inhibitory synapses without affecting the overall number of vesicles. We detected no difference in the density of inhibitory inputs. These findings provide a novel mechanism for the functional effects of estrogen on synaptic inhibition and represent the first in vivo evidence that the number of presynaptic vesicles available for release is a regulated property of synapses that affects synaptic physiology.

Key words: estrogen; hippocampus; GABA; IPSC; paired-pulse depression; serial reconstruction

\section{Introduction}

Synaptic transmission depends on neurotransmitter release from vesicles that are docked at the presynaptic membrane and primed for membrane fusion and exocytosis (Sudhof, 1995). Presynaptic action potentials evoke transmitter release with a probability less than 1 . Although many factors can influence probability of release, studies of excitatory synapses in the hippocampus indicate that release probability is positively related to the size of a readily releasable pool of vesicles (Dobrunz and Stevens, 1997). At hippocampal synapses in culture (Schikorski and Stevens, 2001) and ribbon synapses on retinal bipolar cells (Von Gersdorff et al., 1996), vesicles closely aligned to the presynaptic membrane correspond to the readily releasable pool. In the hippocampus, both release probability (Hessler et al., 1993; Rosenmund et al., 1993) and docked vesicle number (Harris and Sultan, 1995) are highly variable, and the statistical distribution of docked vesicle pool sizes corresponds well with the distribution of release probabilities (Schikorski and Stevens, 1997). A similar relationship is also apparent at excitatory synapses in the cortex (Bower and Haberly, 1986; Schikorski and Stevens, 1999).

Despite these correlations, at least two studies challenge the view that the number of docked vesicles parallels release probability. Ultrastructural comparisons of synapse types with very different release probabilities, parallel fiber versus climbing fiber

Received Aug. 24, 2004; revised Dec. 8, 2004; accepted Dec. 13, 2004.

This work was supported by National Institutes of Health Grants NS 37324 and MH 067564, the W. M. Keck Foundation, and the Northwestern University Biological Imaging Facility. We thank Dr. Indira Raman for helpful comments on this manuscript.

Correspondence should be addressed to Catherine S. Woolley, Department of Neurobiology and Physiology, 2205 Tech Drive, Northwestern University, Evanston, IL60208. E-mail: cwoolley@northwestern.edu.

DOI:10.1523/JNEUROSCI.3489-04.2005

Copyright $\odot 2005$ Society for Neuroscience $\quad$ 0270-6474/05/250971-06\$15.00/0 inputs to cerebellar Purkinje cells (Xu-Friedman et al., 2001), and phasic versus tonic firing motor neurons in the crayfish (Millar et al., 2002) show either no difference or an inverse relationship between release probability and number of docked vesicles.

As an alternative approach to address the relationship between presynaptic vesicles and synaptic function, we compared one type of synapse in two hormonal states with known physiological differences. Previously, we showed that $24 \mathrm{~h}$ of estrogen treatment disinhibits hippocampal CA1 pyramidal cells by suppressing GABAergic synaptic transmission. Estrogen decreases the amplitude of evoked IPSCs, decreases the frequency of miniature IPSCs (mIPSCs) (Rudick and Woolley, 2001), decreases paired-pulse depression of IPSCs, and facilitates action potential firing (Rudick et al., 2003). Because in the same studies we found no decrease in mIPSC amplitude, it is unlikely that the effects of estrogen result from decreased postsynaptic responsiveness to GABA. Rather, the electrophysiological data point to either or both of two changes in inhibitory synapses: a structural decrease in the number/density of inhibitory inputs and/or some change in inhibitory synaptic boutons that limits GABA release.

To investigate these possibilities, we analyzed axosomatic inhibitory synapses on CA1 pyramidal cells in estrogen-treated and control rats using serial reconstruction electron microscopy. Our results show that estrogen-induced disinhibition is paralleled by a profound and specific decrease in the number of synaptic vesicles adjacent to the presynaptic membrane (i.e., those in position to be released). We found no decrease in the density of inhibitory inputs. These findings provide the first in vivo evidence that the number of vesicles available for release is a regulated property of synapses that influences synaptic physiology and support the idea that release probability is related to the size of a releasable vesicle pool at hippocampal inhibitory synapses. 


\section{Materials and Methods}

Hormone treatment and tissue processing. The Northwestern University Animal Care and Use Committee approved all of the animal procedures. Eight adult female Sprague Dawley rats (180-220 g) were ovariectomized (OVX) under ketamine $(85 \mathrm{mg} / \mathrm{kg}$, i.p. $)$ and xylazine $(13 \mathrm{mg} /$ $\mathrm{kg}$, i.p.) anesthesia. Three days later, four rats received a $10 \mu \mathrm{g}$ subcutaneous injection of $17 \beta$ estradiol benzoate $(\mathrm{OVX}+\mathrm{E})$ in sesame oil vehicle, and the other four received oil alone $(\mathrm{OVX}+\mathrm{O})$. Twenty-four hours later, the animals were anesthetized with Nembutal $(80 \mathrm{mg}$ / $\mathrm{kg}$, i.p.) and perfused with heparinized saline, followed by $2.5 \%$ paraformaldehyde $/ 0.5 \%$ glutaraldehyde in $0.1 \mathrm{~m}$ phosphate buffer, $\mathrm{pH}$ 7.4. After perfusion, brains were postfixed overnight at $4^{\circ} \mathrm{C}$. Dorsal hippocampal blocks were cut at $100 \mu \mathrm{m}$, stained for $1 \mathrm{~h}$ with $1 \%$ osmium tetroxide, dehydrated in graded ethanols, flat embedded in epoxy resin, and trimmed to contain CA1. Between 60 and 130 serial thin $(\sim 80$ $\mathrm{nm}$ ) sections per block were collected on Formvar-coated slot grids.

Separate sets of sections were collected on nickel grids for postembedding immunogold labeling for GABA. Periodic acid/sodium periodate-etched sections were incubated in 1:500 rabbit anti-GABA IgG (Sigma, St. Louis, MO) for $2 \mathrm{~h}$, followed by 1:20 goat anti-rabbit IgG-20 nm colloidal gold (Ted Pella, Redding, CA) for $1 \mathrm{~h}$. All of the sections were counterstained with $3 \%$ uranyl acetate, followed by $2.66 \%$ Reynold's lead citrate, and then examined with a JEOL (Peabody, MA) 100CXII electron microscope. Tissue was coded so that investigators were blind to hormone treatment conditions until all of the reconstructions and analyses were complete.

Image acquisition and analysis. For each brain, one $600 \times$ image in the middle of a series was used to randomly select four CA1 pyramidal cells for analysis. For each section, two to three micrographs at $10,000 \times$ were taken per cell; negatives were scanned ( 1600 dots per inch) and montaged to form a $16-20 \mu \mathrm{m}$ perimeter of the cell membrane. This was repeated through 60-80 serial sections, and then images were aligned using SEM Align (K. M. Harris, Medical College of Georgia, Augusta, GA and J. C. Fiala, Boston University, Boston, MA; www.synapses.mcg.edu). For each section, the somatic membrane, apposed glial profiles, boutons forming symmetric synapses and/or containing pleiomorphic vesicles, presynaptic densities, and presynaptic vesicles were traced and quantified in IGL Trace (www.synapses.mcg.edu). Synapses were identified as apposed electron-dense regions on bouton and soma membrane having at least three vesicles in the bouton within $50 \mathrm{~nm}$ of the presynaptic membrane. Each measured variable was averaged to obtain a single mean value per animal ( $n=4$ for all of the comparisons). We used unpaired, two-tailed Student's $t$ tests to compare means, Pearson's test for correlations, and the Kolmogarov-Smirnov test to compare distributions. All of the data are presented as mean \pm SEM. Illustrative images were made using $3 \mathrm{D}$ Studio Max 4 (Autodesk, San Raphael, CA).

\section{Results}

We used serial-section electron microscopy to investigate two mechanisms for estrogen-induced disinhibition of hippocampal CA1 pyramidal cells: a decrease in the number of inhibitory boutons in synaptic contact with pyramidal cells and/or a decrease in GABA release at individual synapses. We focused on axosomatic inhibitory synapses because inhibitory innervation of CA1 pyramidal cells is most concentrated at the soma (Megias et al., 2001), and our previous electrophysiological recordings were made with stimulating electrodes placed in the cell body layer very near recorded cells.

Before analyzing synaptic inputs in serial sections, we first confirmed that we could reliably identify GABAergic boutons based on two morphological criteria: pleiomorphic vesicles and symmetric presynaptic and postsynaptic densities. In sections labeled for GABA using postembedding immunogold (Fig. $1 \mathrm{~A}$ ), we found that 247 of 259 (95\%) axosomatic boutons (184 of 192 $\mathrm{OVX}+\mathrm{O}$ and 63 of $67 \mathrm{OVX}+\mathrm{E}$ ) that fulfilled both criteria were also GABA positive. Given that we could identify the vast majority of GABAergic boutons morphologically, we proceeded with serial reconstruction using nonimmunolabeled tissue because procedures for immunolabeling compromise ultrastructure quality.

\section{Estrogen does not affect glial ensheathment or density of inhibitory inputs}

Initially, we favored the hypothesis that estrogen-induced disinhibition is attributable to a decrease in inhibitory synapse number because of a precedent in the arcuate nucleus of the hypothalamus. Perez et al. (1993) reported that $24 \mathrm{~h}$ of estrogen treatment (same as that used here) produces hypertrophy of the glial cells that ensheath arcuate neurons, physically displacing axosomatic GABAergic inputs from their postsynaptic sites and increasing arcuate neuron firing (Parducz et al., 2002).

To evaluate glial hypertrophy and axosomatic bouton displacement in the hippocampus, we reconstructed patches of CA1 pyramidal cell body membrane, as well as glial processes and inhibitory boutons apposed to each patch $($ Fig. $1 B)$. Patches from four cells in each of four OVX $+\mathrm{O}$ and four OVX $+\mathrm{E}$ animals were 
Table 1. Ultrastructural characteristics of axosomatic boutons and symmetric synapses

\begin{tabular}{|c|c|c|c|c|}
\hline & $\begin{array}{l}0 \mathrm{VX}+0 \\
(n=4)\end{array}$ & $\begin{array}{l}0 \mathrm{VX}+\mathrm{E} \\
(n=4)\end{array}$ & $p$ values & $\begin{array}{l}\text { Combined } \\
(n=8)\end{array}$ \\
\hline \multicolumn{5}{|l|}{ Bouton volume (all } \\
\hline boutons; $\mu \mathrm{m}^{3}$ ) & $0.37 \pm 0.04$ & $0.31 \pm 0.03$ & 0.23 & $0.34 \pm 0.02$ \\
\hline \multicolumn{5}{|l|}{ Bouton volume } \\
\hline $\begin{array}{l}\text { (with mitochon- } \\
\text { dria; } \mu \mathrm{m}^{3} \text { ) }\end{array}$ & $0.45 \pm 0.05$ & $0.38 \pm 0.04$ & 0.25 & $0.41 \pm 0.03$ \\
\hline \multicolumn{5}{|l|}{ Bouton volume } \\
\hline $\begin{array}{l}\text { (lacking mito- } \\
\text { chondria; } \mu \mathrm{m}^{3} \text { ) }\end{array}$ & $0.12 \pm 0.02$ & $0.11 \pm 0.04$ & 0.73 & $0.12 \pm 0.02$ \\
\hline \multicolumn{5}{|l|}{ Vesicle density } \\
\hline$\left(\# / \mu \mathrm{m}^{3}\right)$ & $1654 \pm 139$ & $1301 \pm 198$ & 0.20 & $1477 \pm 130$ \\
\hline $\begin{array}{l}\text { Boutons with mito- } \\
\text { chondria (\%) }\end{array}$ & \multicolumn{3}{|c|}{ Boutons with mito- } & $76 \pm 2$ \\
\hline \multicolumn{5}{|l|}{ Presynaptic density } \\
\hline area $\left(\mu \mathrm{m}^{2}\right)$ & $0.10 \pm 0.02$ & $0.07 \pm 0.01$ & 0.20 & $0.08 \pm 0.0$ \\
\hline \multicolumn{5}{|l|}{ Multiple synapse } \\
\hline boutons (\%) & $34 \pm 4$ & $36 \pm 5$ & 0.72 & $35 \pm 1$ \\
\hline
\end{tabular}

reconstructed from 60-80 serial electron micrographs per cell. Each patch was $\sim 75 \mu \mathrm{m}^{2}$ in area, or approximately one-fifth of the total somatic surface. From these reconstructions, we quantified the percentage of somatic surface apposed by glia, the density of synaptically connected inhibitory boutons, and the distance from each inhibitory synaptic density to the nearest glial profile. None of these parameters was significantly affected by estrogen. There was no evidence that estrogen produces hypertrophy of somatic glial ensheathment because the percentage of somatic area apposed by glia was $26.8 \pm 2.7 \%$ on OVX $+\mathrm{O}$ cells and $30.2 \pm 1.8 \%$ on OVX $+\mathrm{E}$ cells $(p=0.33)$ (Fig. $1 C)$. Additionally, there was no evidence that estrogen decreases axosomatic inhibitory innervation; OVX $+\mathrm{O}$ and $\mathrm{OVX}+\mathrm{E}$ cells had similar densities of synaptically connected inhibitory boutons. Connected bouton density was $0.16 \pm 0.02$ boutons $/ \mu \mathrm{m}^{2}$ on $\mathrm{OVX}+\mathrm{O}$ cells and $0.19 \pm 0.02$ boutons $/ \mu \mathrm{m}^{2}$ on OVX $+\mathrm{E}$ cells $(p=0.40)$ (Fig. $1 D)$. Finally, we found no evidence of a change in the spatial relationship between glia and inhibitory synapses. The average distance between an inhibitory synapse and the nearest glial profile was $0.93 \pm 0.27 \mu \mathrm{m}$ for OVX $+\mathrm{O}$ cells and $0.70 \pm$ $0.07 \mu \mathrm{m}$ for OVX + E cells ( $p=0.44$; data not shown). Together, these data effectively refuted the hypothesis that glial hypertrophy and axosomatic bouton displacement underlie estrogeninduced disinhibition of CA1 pyramidal cells.

\section{Estrogen decreases the number of vesicles adjacent to the presynaptic membrane}

Subsequently, we considered the possibility that estrogen produces a change in presynaptic boutons that could limit GABA release. A total of 373 axosomatic boutons forming 510 inhibitory synapses (OVX $+\mathrm{O}, 166$ boutons forming 219 synapses; OVX $+\mathrm{E}$, 207 boutons forming 291 synapses) that were completely contained within our series were used to quantify bouton volume, presynaptic density area, and vesicle number, density, and location. Boutons contained only partially within a series were excluded. The number of synapses is greater than the number of boutons because some boutons form multiple synapses. With the exception of vesicle location (see below), none of the measured parameters was significantly affected by estrogen (Table 1). As observed for excitatory synapses in the hippocampus (Harris and
Sultan, 1995; Schikorski and Stevens, 1997), bouton volume was positively correlated with vesicle number $(r=0.86 ; p<0.001)$ and size of the postsynaptic density $(r=0.56 ; p<0.001)$. Most inhibitory boutons $(76 \%)$ contained mitochondria; boutons containing mitochondria were $\sim 3.4$ times larger than boutons not containing them (Table 1 ).

In contrast to other parameters examined, analysis of vesicle location revealed that estrogen produced a profound decrease in the number of vesicles adjacent to the presynaptic density. For each synapse, we quantified the number of vesicles located $0-30$, $31-50$, or $\geq 51 \mathrm{~nm}$ from the presynaptic membrane. We chose 30 $\mathrm{nm}$ as the limit of the closest range because this is the shortest distance from a synapse that we could measure reliably. Because our experimental design involved the evaluation of all of the synapses within a particular volume of tissue, some synapses or parts of synapses were sectioned obliquely; in these cases, it was not possible to confirm all of the vesicles that were physically associated with the presynaptic membrane. However, this distance range is within that reported by others to reflect docked vesicles [e.g., 0-70 nm (Schikorski and Stevens, 2001)], and it is likely that the vast majority of vesicles $0-30 \mathrm{~nm}$ from the presynaptic membrane belong to a releasable vesicle pool (Stanton et al., 2003).

Reconstructed synapses from OVX $+\mathrm{O}$ (Fig. 2A,C) and $\mathrm{OVX}+\mathrm{E}$ (Fig. $2 B, D$ ) animals were used to quantify the number of vesicles within $30 \mathrm{~nm}$ of the presynaptic membrane (Fig. $2 \mathrm{E}-$ $H)$. As has been shown for excitatory synapses, the number of vesicles near the presynaptic membrane at inhibitory synapses was highly variable, ranging from 0 to 61 . Interestingly, estrogentreated animals had more than twice as many synapses with no vesicles within $30 \mathrm{~nm}$, although this was a small proportion of synapses overall. OVX $+\mathrm{E}$ animals had $13.8 \pm 1.7 \%$ synapses with zero vesicles within $30 \mathrm{~nm}$ compared with $5.6 \pm 1.8 \%$ in $\mathrm{OVX}+\mathrm{O}$ animals $(p<0.01)$ (Fig. $2 E, F)$. Among synapses that did contain vesicles within $0-30 \mathrm{~nm}$, the distribution was significantly shifted toward fewer vesicles in OVX $+\mathrm{E}$ animals $(p<$ 0.01 ) (Fig. 2G). This shift led to, on average, one-half as many vesicles adjacent to the presynaptic membrane in estrogentreated animals compared with controls. OVX $+\mathrm{E}$ animals had $6.2 \pm 1.3$ vesicles within $30 \mathrm{~nm}$ of the presynaptic membrane, whereas $\mathrm{OVX}+\mathrm{O}$ animals had $12.1 \pm 1.2$ vesicles within this range $(p=0.01)$ (Fig. $2 H$ ). This decrease in number of vesicles near the synapse was paralleled by a decrease in vesicle density. The density of vesicles from 0 to $30 \mathrm{~nm}$ was $440 \pm 74$ vesicles/ $\mu \mathrm{m}^{3}$ in OVX $+\mathrm{E}$ compared with $828 \pm 95$ vesicles $/ \mu \mathrm{m}^{3}$ in $\mathrm{OVX}+\mathrm{O}(p=0.02)$. Slightly farther from the synapse, 31-50 $\mathrm{nm}$, the average number of vesicles was lower in OVX $+\mathrm{E}$ animals (15.6 \pm 3.4$)$ compared with OVX $+\mathrm{O}$ animals $(24.1 \pm 4.5)$, but this difference was not statistically significant $(p=0.19)$. Similarly, the number of vesicles $\geq 51 \mathrm{~nm}$ from the presynaptic density was not significantly different between OVX $+\mathrm{E}(525 \pm 109)$ and $\mathrm{OVX}+\mathrm{O}(763 \pm 134)$ animals $(p=0.22)$. Thus, estrogen specifically decreases the number of vesicles adjacent to the presynaptic membrane of axosomatic inhibitory synapses in CA1 without significantly affecting the overall number of vesicles or any other structural parameter in inhibitory boutons.

\section{Discussion}

The results presented here show that estrogen-induced disinhibition of hippocampal CA1 pyramidal cells is associated with a decrease in the number of vesicles adjacent to the presynaptic membrane of inhibitory synapses (i.e., those vesicles most likely to be readily releasable). A reduction in the number of releasable 
vesicles provides a novel mechanism for the functional effects of estrogen on GABAergic inhibition. Each of the previously established electrophysiological effects of estrogen is consistent with a reduction in GABA release: decreased amplitude of evoked IPSCs, decreased frequency, but not amplitude, of mIPSCs (Rudick and Woolley, 2001), and decreased paired pulsedepression of IPSCs (Rudick et al., 2003). Because estrogen did not affect the density of inputs, our findings indicate that estrogeninduced disinhibition in the hippocampus occurs via a different mechanism than it does in the hypothalamus, in which estrogen has been reported to decrease the density of inhibitory inputs to arcuate neurons (Perez et al., 1993).

To our knowledge, this is the first demonstration in vivo that the number of neurotransmitter vesicles available for release is a regulated property of synapses that affects synaptic physiology. Studies in the 1970s and 1980s showed that sensory or electrical stimulation in vivo can acutely alter the distribution of presynaptic vesicles (Feher et al., 1972; Applegate and Landfield, 1988). Subsequently, Murthy et al. (2001) found that pharmacological blockade of activity in hippocampal cultures increases the number of releasable presynaptic vesicles. However, none of these studies addressed whether manipulations other than the modulation of activity affects vesicle distribution or whether the distribution of vesicles is regulated in a way that leads to a stable change in synaptic function. Tyler and Pozzo-Miller (2001) did investigate these questions in hippocampal slice cultures. These investigators found that culturing slices with exogenous brain-derived neurotrophic factor increased both the number of vesicles docked at excitatory synapses and the frequency, but not amplitude, of miniature EPSCs (mEPSCs). This coordinated change in the number of docked vesicles and mEPSC frequency in cultures mirrors what we found for the effects of estrogen on inhibitory synapses in the adult brain (although in the opposite direction). That releasable vesicle number and mEPSC/mIPSC frequency change in parallel under two such different circumstances strongly suggests a causal relationship.

\section{Release probability, paired-pulse depression, and the docked vesicle pool}

Release probability is a critical variable in synaptic function. One way to assess release probability is with paired-pulse experiments, in which two synaptic stimuli are given in close succession and the postsynaptic response evoked by the second pulse is compared with that evoked by the first. At GABAergic synapses on CA1 pyramidal cells, the second response tends to be depressed compared with the first (Davies et al., 1990), and the amount of depression can be increased by increasing initial release probability (Jiang et al., 2000).

On one hand, this relationship between paired-pulse depression and release probability might be explained by depletion of release sites. For example, if, after releasing a single vesicle, a release site becomes refractory until some repriming event occurs, then the pool of potential release sites would be depleted temporarily. Thus, when the probability is high that any site will release a vesicle, a greater fraction of sites releases on the first stimulus, leaving relatively fewer sites capable of release on the second stimulus, leading to depression. Conversely, when release probability is low, less depression is observed because a relatively greater fraction of release sites remains capable of release on the second stimulus. Given the positive relationship between the number of docked vesicles and release probability shown for hippocampal excitatory synapses (Dobrunz and Stevens, 1997; Schikorski and Stevens, 2001), this depletion model predicts that a smaller pool of docked vesicles at each release site should be associated with lower release probability and therefore less pairedpulse depression.

On the other hand, the situation is not necessarily so simple. Two previous serial electron microscopic studies that compared docked vesicle number at different types of synapses with high versus low release probability and correspondingly different behavior in paired-pulse experiments showed either no difference (Xu-Friedman et al., 2001) or the opposite of that predicted by a depletion model (Millar et al., 2002). This may be because variables in addition to the number of releasable vesicles can influence release probability, including $\mathrm{Ca}^{2+}$ influx, $\mathrm{Ca}^{2+}$ buffering, and $\mathrm{Ca}^{2+}$ sensitivity of release machinery (for review, see Zucker and Regehr, 2002). Furthermore, a relationship between release probability and paired-pulse depression can be complicated by factors such as postsynaptic receptor desensitization (Kirischuk et al., 2002), negative feedback via presynaptic $\mathrm{GABA}_{\mathrm{B}}$ receptors (Lambert and Wilson, 1994), interactions between release sites (Telgkamp et al., 2004), or processes upstream of exocytosis (Kraushaar and Jonas, 2000). If factors such as these differ between the synapses compared in previous electron microscopic studies, they could have obscured an underlying relationship between docked vesicle number and release probability.

Our findings add a new piece to this complex puzzle and support the idea that release probability, paired-pulse depression, 
and the docked vesicle pool are directly related. The approach was to evaluate the same type of synapse in two hormonal states with known differences in physiology. Previous studies established that the same estrogen treatment used here decreases the amplitude of IPSCs evoked by single stimuli and reduces paired-pulse depression. Together, these data suggest a scenario in which an estrogen-induced decrease in the number of vesicles available for release at inhibitory synapses reduces release probability, resulting in GABA release from fewer release sites and, consequently, smaller evoked IPSCs and less depression of the second IPSC in paired-pulse experiments. Estrogen may also affect nonstructural parameters that influence release probability and/or synaptic depression. However, whether or not such additional effects exist, our data suggest a model in which release probability at inhibitory synapses in CA1 is related to the size of the releasable pool of vesicles, and depletion of release sites could contribute to synaptic depression.

\section{Estrogen action in the hippocampus}

In addition to its effects on inhibitory synapses, estrogen also regulates excitatory synapses in CA1. Seventy-two hours of estrogen treatment increases the density of dendritic spines and excitatory synapses on CA1 pyramidal cells, increases the sensitivity of CA1 pyramidal cells to excitatory synaptic input, enhances long-term potentiation (for review, see Woolley, 1998), and improves spatial working memory (Sandstrom and Williams, 2001). Based on parallels with the effects of estrogen on dendritic spines in vitro (Murphy et al., 1998), estrogen-induced suppression of inhibitory synaptic transmission is likely to play a causal role in subsequent increases in spine and synapse density. Therefore, understanding how estrogen regulates synaptic inhibition is key to understanding estrogen-induced synaptogenesis in the hippocampus.

The classical mechanism of estrogen action is via nuclear transcription factor receptors. A curious aspect of the effects of estrogen in the dorsal hippocampus is the paucity of cells that express nuclear estrogen receptors (ERs). The few cells in dorsal CA1 that do express nuclear ERs are GABAergic neurons, but this is only a small subset of all of the GABAergic neurons in the region (Hart et al., 2001). Interestingly, extranuclear ER immunoreactivity has also been detected in some axons and glia in CA1 (Milner et al., 2001), particularly those in the pyramidal cell layer (S. A. Hart and C. S. Woolley, unpublished observations). Because glia have been shown to regulate GABA release from CA1 interneurons (Kang et al., 1998), it is tempting to speculate that ER signaling via an extranuclear mechanism in glia and/or presynaptic boutons could be involved in the effects of estrogen on synaptic inhibition of pyramidal cells. Whether the estrogen-induced decrease in vesicles adjacent to the presynaptic membrane results from vesicle depletion after an initial stimulation of release and/or slower refilling of release sites is unknown. In either case, regulation of inhibitory presynaptic vesicle dynamics reflects a novel estrogen action in the hippocampus that influences inhibitory synaptic physiology and may also play a role in subsequent excitatory synaptogenesis.

\section{References}

Applegate MD, Landfield PW (1988) Synaptic vesicle redistribution during hippocampal frequency potentiation and repression in young and aged rats. J Neurosci 8:1096-1111.

Bower JM, Haberly LB (1986) Facilitating and nonfacilitatiing synapses on pyramidal cells: a correlation between physiology and morphology. Proc Natl Acad Sci USA 83:1115-1119.
Davies CH, Davies SN, Collingridge GL (1990) Paired-pulse depression of monosynaptic GABA-mediated inhibitory postsynaptic responses in rat hippocampus. J Physiol (Lond) 424:513-531.

Dobrunz LE, Stevens CF (1997) Heterogeneity of release probability, facilitation, and depletion at central synapses. Neuron 18:995-1008.

Feher O, Joo F, Halasz N (1972) Effect of stimulation on the number of synaptic vesicles in the nerve fibers and terminals of the cerebral cortex in the cat. Brain Res 47:37-48.

Harris KM, Sultan P (1995) Variation in the number, location and size of synaptic vesicles provides an anatomical basis for the nonuniform probability of release at hippocampal CA1 synapses. Neuropharmacology 34:1387-1395.

Hart SA, Patton JD, Woolley CS (2001) Quantitative analysis of ER $\alpha$ and GAD colocalization in the hippocampus of the adult female rat. J Comp Neurol 440:144-155.

Hessler NA, Shirke AM, Malinow R (1993) The probability of transmitter release at a mammalian central synapse. Nature 366:569-572.

Jiang L, Sun S, Nedergaard M, Kang J (2000) Paired-pulse modulation at individual GABAergic synapses in rat hippocampus. J Physiol (Lond) 523:425-439.

Kang J, Goldman SA, Nedergaard M (1998) Astrocyte-mediated potentiation of inhibitory synaptic transmission. Nat Neurosci 1:683-692.

Kirischuk S, Clements JD, Grantyn R (2002) Presynaptic and postsynaptic mechanisms underlie paired pulse depression at single GABAergic boutons in rat collicular cultures. J Physiol (Lond) 543:99-116.

Kraushaar U, Jonas P (2000) Efficacy and stability of quantal GABA release at a hippocampal interneuron-principal neuron synapse. J Neurosci 20:5594-5607.

Lambert NA, Wilson WA (1994) Temporally distinct mechanisms of usedependent depression at inhibitory synapses in the rat hippocampus in vitro. J Neurophysiol 72:121-130.

Megias M, Emri Z, Freund TF, Gulyas AI (2001) Total number and distribution of inhibitory and excitatory synapses on hippocampal CA1 pyramidal cells. Neuroscience 102:527-540.

Millar AG, Bradacs H, Charlton MP, Atwood HL (2002) Inverse relationship between release probability and readily releasable vesicles in depressing and facilitating synapses. J Neurosci 22:9661-9667.

Milner TA, McEwen BS, Hayashi S, Li CJ, Reagan LP, Alves SE (2001) Ultrastructural evidence that hippocampal alpha estrogen receptors are located at extranuclear sites. J Comp Neurol 429:355-371.

Murphy DD, Cole NB, Greenberger V, Segal M (1998) Estradiol increases dendritic spine density by reducing GABA neurotransmission in hippocampal neurons. J Neurosci 18:2550-2559.

Murthy VN, Schikorski T, Stevens CF, Zhu Y (2001) Inactivity produces increases in neurotransmitter release and synapse size. Neuron 32:673-682.

Parducz A, Hoyk Z, Kis Z, Garcia-Segura LM (2002) Hormonal enhancement of neuronal firing is linked to structural remodelling of excitatory and inhibitory synapses. Eur J Neurosci 16:665-670.

Perez J, Luquin S, Naftolin F, Garcia-Segura LM (1993) The role of estradiol and progesterone in phased synaptic remodeling of the rat arcuate nucleus. Brain Res 608:38-44.

Rosenmund C, Clements JD, Westbrook GL (1993) Nonuniform probability of glutamate release at a hippocampal synapse. Science 262: $754-757$.

Rudick CN, Woolley CS (2001) Estrogen regulates functional inhibition of hippocampal CA1 pyramidal cells in the adult female rat. J Neurosci 21:6532-6543.

Rudick CN, Gibbs RB, Woolley CS (2003) A role for the basal forebrain cholinergic system in estrogen-induced disinhibition of hippocampal pyramidal cells. J Neurosci 23:4479-4490.

Sandstrom NJ, Williams CL (2001) Memory retention is modulated by acute estradiol and progesterone replacement. Behav Neurosci 115:384-393.

Schikorski T, Stevens CF (1997) Quantitative ultrastructural analysis of hippocampal excitatory synapses. J Neurosci 17:5858-5867.

Schikorski T, Stevens CF (1999) Quantitative fine-structural analysis of olfactory cortical synapses. Proc Natl Acad Sci USA 96:4107-4112.

Schikorski T, Stevens CF (2001) Morphological correlates of functionally defined synaptic vesicle populations. Nat Neurosci 4:391-395. 
Stanton PK, Winterer J, Bailey CP, Kyrozis A, Raginov I, Laube G, Veh RW, Nguyen CQ, Muller W (2003) Long-term depression of presynaptic release from the readily releasable vesicle pool induced by NMDA receptor-dependent retrograde nitric oxide. J Neurosci 23:5936-5944.

Sudhof TC (1995) The synaptic vesicle cycle: a cascade of protein-protein interactions. Nature 375:645-653.

Telgkamp P, Padgett DE, Ledoux VA, Woolley CS, Raman IR (2004) Maintenance of high-frequency transmission at Purkinje to cerebellar nuclear synapses by spillover from boutons with multiple release sites. Neuron 41:113-126.

Tyler WJ, Pozzo-Miller LD (2001) BDNF enhances quantal neurotrans- mitter release and increases the number of docked vesicles at the active zones of hippocampal excitatory synapses. J Neurosci 21:4249-4258.

Von Gersdorff H, Vardi E, Matthews G, Sterling P (1996) Evidence that vesicles on the synaptic ribbon of retinal bipolar neurons can be rapidly released. Neuron 16:1221-1227.

Woolley CS (1998) Estrogen-mediated structural and functional synaptic plasticity in the female rat hippocampus. Horm Behav 34:140-148.

Xu-Friedman MA, Harris KM, Regehr WG (2001) Three-dimensional comparison of ultrastructural characteristics at depressing and facilitating synapses onto cerebellar Purkinje cells. J Neurosci 21:6666-6672.

Zucker RS, Regehr WG (2002) Short-term synaptic plasticity. Annu Rev Physiol 64:355-405. 